\title{
Células madre mesenquimales: características biológicas y aplicaciones clínicas.
}

\author{
Jenny Andrea Arévalo Romero. Bcl, Diana Marcela Páez Guerrero. Bcl \\ Viviana Marcela Rodríguez Pardo. Bcl. MSc
}

Laboratorio de Hematología Pontificia Universidad Javeriana

Correspondencia: vivianar@javeriana.edu.co, vivianarodrigu@gmail.com

Recibido: 04-10-07 / Aceptado: 10-11-07

\begin{abstract}
Resumen
Las células madre mesenquimales son células pluripotentes y adultas con morfología fibroblastoide y plasticidad hacia diversos linajes celulares como condrocitos, osteocitos y adipocitos entre otros. Estas células pueden ser aisladas principalmente de médula ósea, sangre de cordón umbilical y tejido adiposo de donde se han logrado establecer cultivos que han permitido estudiar sus propiedades funcionales y fenotípicas. Aunque la información obtenida hasta la fecha no brinda un conocimiento completo, se espera que con el desarrollo de próximas investigaciones se aclaren diversos aspectos biológicos para implementar su uso en medicina regenerativa. Esta revisión presenta una visión general sobre las células madre mesenquimales: morfología e inmunofenotipo, ontogenia, fuentes de obtención y aplicaciones clínicas.
\end{abstract}

Palabras claves: célula madre, diferenciación celular, mesenquimal, fibroblastos, médula ósea, osteocitos.

\begin{abstract}
Mesenchymal stem cells are pluripotent and adult with fibroblastoid morphology and plasticity towards different cell lineages such as chondrocytes, osteocytes, and adipocytes among others. These cells can be isolated mainly from bone marrow, umbilical cord blood, and adipose tissue where it has been possible to establish cultures that have allowed the study of their functional and phenotypic properties. Although the information that has been collected thus far does not offer a complete knowledge, it is hoped that with future research several biological aspects can be cleared up to implement its use in regenerative medicine. This revision presents a general vision of mesenchymal stem cells, morphology and immunophenotype, ontogeny, sources, and clinical applications.
\end{abstract}

Key Words: bone marrow, cell differentiation, fibroblast, mesenchymal stem cell, osteocytes, stem cell.

\section{Morfología e inmunofenotipo.}

Los progenitores mesenquimales son un grupo de células madres adultas que fueron caracterizadas por Friedenstein, quien las aisló de médula ósea y las describió como células adherentes de morfología fibroblastoide, capaces de diferenciarse hacia células de origen mesodérmico como osteocitos, condrocitos y adipocitos (1- 4). Varios estudios han asignado a este grupo celular diversos nombres como: Células de Estroma Medular, Unidades Formadoras de Colonias Fibroblastoides, Precursores Estromales ó Células Adultas Progenitoras Multipotentes o MAPCs (Multi-Potent Adult Progenitor 
Cells). En el año 2006, la Sociedad Internacional de Terapia Celular ó ISCT (Internacional Society Cellular Therapy) propuso tres criterios para definir las células madre mesenquimales (CMM); primero, éstas células deben ser adherentes en cultivo; segundo, expresar los antigenos CD73, CD90 y CD105 en ausencia de antigenos hematopoyéticos como CD34, CD45, marcadores de monocitos, macrófagos y linfocitos B; y tercero, las CMM deben ser capaces de diferenciarse in vitro en osteoblastos, adipocitos y condrocitos bajo condiciones estándar de cultivo $(2,5-8)$.

Además de lo propuesto por ISCT, también se debe tener en cuenta dos aspectos adicionales para clasificarlas como células madre: que las CMM realicen procesos de autorenovación, es decir, durante la división celular solo una de las células hijas debe iniciar programas de diferenciación celular y que sean capaces de desarrollar «plasticidad clonogénica» o diferenciación hacia tejidos de diferentes capas embrionarias como ectodermo y endodermo $(9,10)$.

ISCT propone la molécula CD73 o 5, ectonucleotidasa como un marcador de linaje para las CMM. Esta es una glicoproteína cuya función biológica consiste en hidrolizar nucleótidos extracelulares para permitir el ingreso de nucleósidos y así generar ATP y GPT como fuente de energía celular en células diferenciadas. No obstante, el papel que juega CD73 en células madre mesenquimales se cree que está más relacionado con mecanismos de adhesión celular ya que se ha encontrado co-expresada con moléculas tipo â2 integrinas, lo que ha postulado a CD73 como un mediador de adhesión celular en CMM $(13,14)$.

Las CMM, aisladas de médula ósea, también expresan la molécula CD90 ó Thy-1, una proteína que hace parte de la superfamilia de las inmunoglobulinas y cuyo principal ligando es CD45 $(15,16)$; se expresa entre el $10-40 \%$ de las células CD34, y en mayor proporción en tejido conectivo y en células del estroma medular y aunque no se conoce su función en células madre mesenquimales, un estudio muestra que si son sometidas a stress celular mecánico se diferencian hacia células similares a osteoblastos disminuyendo notablemente la expresión de CD90, lo que podría mostrar que este antígeno es un marcador de precursores mesenquimales tempranos que pueden diferenciarse en osteoblastos (17).

CD105, también conocida como endoglina, es una glicoproteína que hace parte del complejo del receptor del Factor Transformante de Crecimiento-B ó $T G F-\hat{a}$ y se expresa en monocitos activados, macrófagos activados, precursores eritroides, fibroblastos, células foliculares dendríticas, melanocitos, células cardiacas, células vasculares de músculo liso, células endoteliales. CD105 interviene en la regulación de distintos componentes de la matriz extracelular como fibronectina y colágeno razón por la cual se cree que está relacionada con procesos de angiogénesis y reparación vascular (18, 19) y se sugiere que su expresión en CMM humanas es determinante en la generación de cardiomiocitos (20).

Además de los antigenos propuestos por ISCT, otros autores proponen moléculas como STRO-1, CD44 y CD166 para la tipificación de células mesenquimales. En 1991 se identificó a STRO-1 como un antígeno específico para CMM aisladas de médula ósea (21). STRO-1 es un marcador que se expresa en el desarrollo temprano de dichas células, declinando su expresión cuando genes asociados a la diferenciación y expansión osteogénica como el Factor de Unión Core A1 (CBFA1) interactúa con osteopontina y osteocalcina (22). CD44 es una molécula de adhesión que actúa mediante la interacción con el ácido hialurónico, osteopontina, colágeno, anquirina, fibronectina y metaloproteinasas (23) y contribuye en procesos de adhesión, migración y proliferación de CMM (24). Además de STRO-1 y CD44, se sugiere que la molécula CD166 o ALCAM (Activated Leukocyte Cell Adhesión Molecule) interviene en la hematopoyesis involucrando a las CMM, participando en el mantenimiento del estado indiferenciado de células madre hematopoyéticas y CMM $(6,25)$. En la Tabla 1 se observan otros marcadores útiles en la identificación de CMM (8). 
T abla 1Proteínas expresadas en células madre mesenquimales.

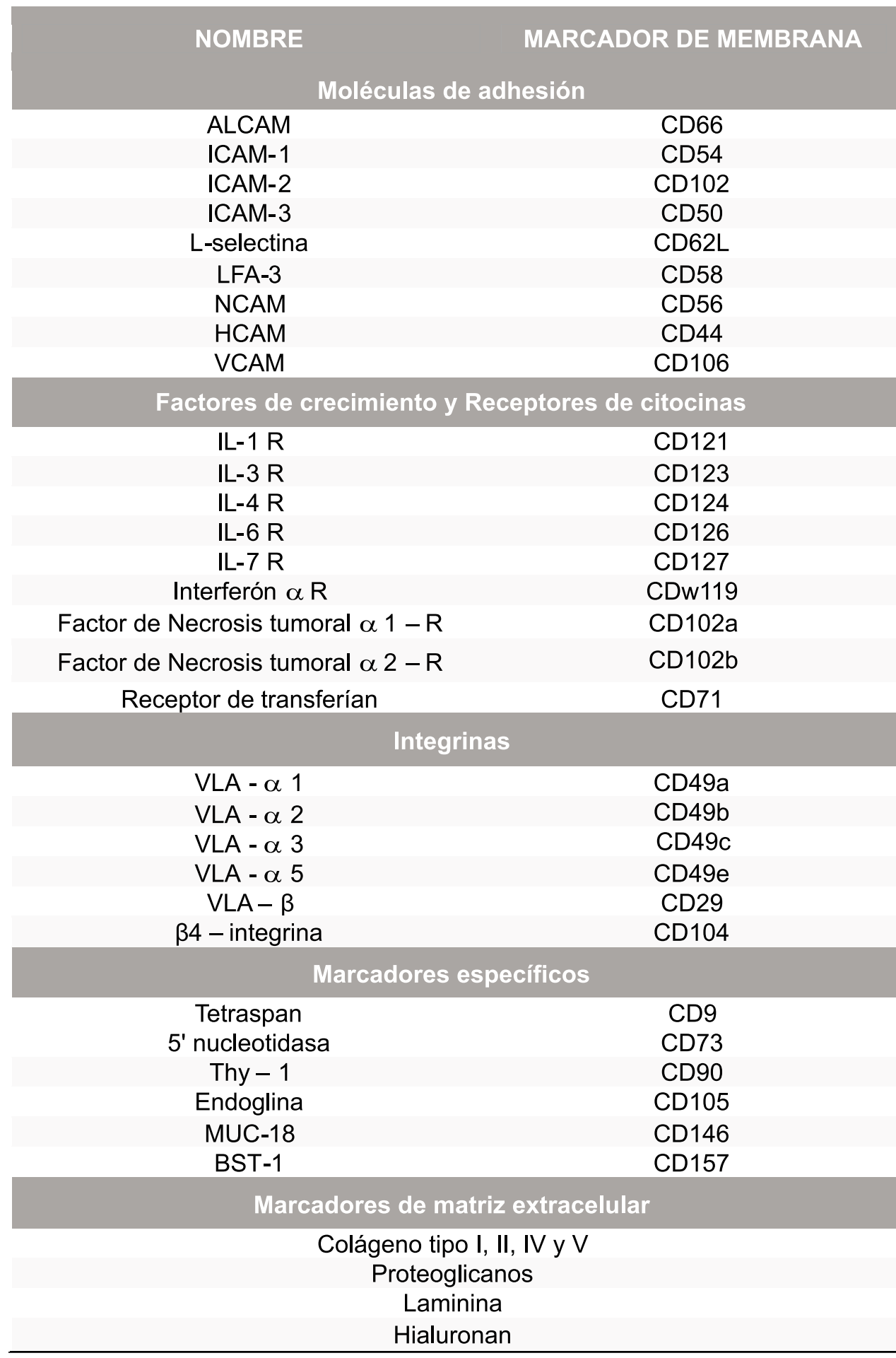

T omado de Deans RJ, Moseley AB. 2000.

Aunque las CMM fueron descritas como células similares a fibroblastos, la morfología de las CMM puede variar según la fuente de obtención; por ejemplo, se ha observado que cuando se aíslan a partir de sangre de cordón umbilical el 93\% de la población celular tiene un aspecto ovoide, con respecto al $7 \%$ que conserva una morfología similar a los fibroblastos (4). Por el contrario, cuando estas células son aisladas a partir de medula ósea 
la relación de células fibroblastoides es mayor con respecto a las ovoides $(4,11)$. Este aspecto morfológico es de gran importancia ya que podría tener relación con el inmunofenotipo de estas células según lo postulado por Chang YJ y colaboradores quienes demuestran que si la población mesenquimal es obtenida de sangre de cordón umbilical la gran mayoría no expresan el antigeno CD90, mientras que si la población es de origen medular este marcador es determinante en su identificación, lo que podría explicar en parte los contradictorios resultados que se encuentran publicados con respecto a la expresión de ésta y otras proteínas en CMM $(4,12)$.

\section{Ontogenia}

Algunos autores sugieren que las CMM tienen un desarrollo embrionario paralelo y coordinado junto con las células madre hematopoyéticas $(\mathrm{CMH})$; lo que se demuestra en embriones de ratón con 11 días de gestación, en los cuales se han detectado CMM en la región aorta-gónada-mesonefro (AGM) donde también aparecen las primeras CMH (29).

Además se ha postulado que algunas regiones embrionarias proveen el microambiente necesario para la diferenciación de CMM, tal es el caso de los surcos urogenitales (UGRs) donde se han encontrado una gran cantidad de progenitores adiposos en comparación con las regiones AGM y la aorta dorsal que rodea el mesénquima (AoM); contrario a esto, los progenitores condrogénicos están presentes en mayor frecuencia en la región AoM. Esto podría mostrar que durante la embriogénesis, las CMM migran a lugares diferentes para realizar procesos de diferenciación celular (29).

\section{Fuentes de obtención}

La médula ósea es la principal fuente de aislamiento de CMM, aunque se han aislado de tejido adiposo, páncreas, hígado, músculo esquelético, dermis, membrana sinovial, hueso trabecular (30-33), sangre de cordón umbilical (34), tejido pulmonar (35), pulpa dental y ligamento periodontal (36). No obstante, los tejidos más empleados son la medula ósea, la sangre de cordón umbilical y el tejido adiposo. Aunque la médula ósea es la mejor fuente de obtención; $0.003 \%$ de las células mononucleares de médula ósea humana son CMM según Beyer N y Da Silva L 2006, existen algunos aspectos que dificultan su uso como: limitada tasa de crecimiento, la capacidad de diferenciación de acuerdo a la edad del donante de la médula y el riesgo en la toma de muestra $(30,32)$.

Sobre la obtención de células madre mesenquimales a partir de sangre de cordón umbilical, éste procedimiento requiere optimizar puntos críticos para lograr un cultivo exitoso, tales como el tiempo de recolección y procesamiento que debe ser inferior a 16 horas, así como el volumen de sangre recolectado igual o superior a 30 $\mathrm{ml}$ (34). Respecto al aislamiento y cultivo a partir de tejido adiposo, las células obtenidas de esta fuente tienen una morfología, fenotipo y capacidad de diferenciación in vitro similar a las obtenidas de médula ósea, tienen una mayor capacidad de proliferación y es posible acceder más fácilmente a muestras de tejido adiposo a través de procedimientos como liposucción o abdominoplastia $(33,38)$. En la Tabla 2 se presentan otros aspectos comparativos de las principales fuentes de obtención de CMM $(32,33)$.

\section{Aplicaciones clínicas de CMM}

En modelos experimentales se ha demostrado que las CMM son capaces de regenerar tejidos deteriorados o lesionados como hueso, cartílago, tejido hepático o miocárdico $(3,38,39)$; y modular reacciones inmunes en colagenopatías, esclerosis múltiple y trasplantes de médula ósea (7).

Una de las principales aplicaciones clínicas de las CMM consiste en la reparación de hueso, demostrado in vivo en ratones y en caninos con defectos cráneo-faciales y defectos de huesos largos mediante la administración directa de CMM, con matrices como hidroxiapatito/fosfato tricálcico (HA/TPC) mostrando resultados satisfactorios 
T abla 2.Comparación fuentes de obtención de células madre mesenquimales

\begin{tabular}{|c|c|c|c|}
\hline PARAMETRO & Médula ósea & $\begin{array}{l}\text { Sangre de cordón } \\
\text { umbilical }\end{array}$ & $\begin{array}{l}\text { Tejido } \\
\text { adiposo }\end{array}$ \\
\hline Éxito de aislamiento & $100 \%$ & $30-34 \%$ & $100 \%$ \\
\hline Formación de monocapa adherente & 4 - 5 días & $2-4$ semanas & 4 - 5 días \\
\hline $\begin{array}{l}\text { UFC-F obtenidas en la monocapa } \\
\text { adherente (número) }\end{array}$ & $83 \pm 61$ & $0.002 \pm 0.004$ & $557 \pm 673$ \\
\hline $\begin{array}{c}\text { Capacidad de diferenciación } \\
\text { osteogénica }\end{array}$ & $71.4 \%$ & $100 \%$ & $78.8 \%$ \\
\hline $\begin{array}{c}\text { Capacidad de diferenciación } \\
\text { adipogénica }\end{array}$ & $100 \%$ & $0 \%$ & $94 \%$ \\
\hline $\begin{array}{l}\text { Capacidad de diferenciación } \\
\text { condrogénica }\end{array}$ & $100 \%$ & $100 \%$ & $100 \%$ \\
\hline Expresión antígenos (\%) & & & \\
\hline CD44 & $97.5 \pm 5.1$ & $99.7 \pm 0.5$ & $99.8 \pm 0.2$ \\
\hline $\begin{array}{l}\text { CD73 } \\
\text { CD90 } \\
\text { CD105 } \\
\text { HLAI }\end{array}$ & $\begin{array}{c}90.0 \pm 20.0 \\
99.1 \pm 2.5 \\
88.1 \pm 7.4 \\
95.2 \pm 6.0\end{array}$ & $\begin{array}{c}99.3 \pm 1.3 \\
97.8 \pm 7.1 \\
72.4 \pm 20.0 \\
94.3+6.8\end{array}$ & $\begin{array}{l}99.6 \pm 0.5 \\
99.6 \pm 0.2 \\
90.4 \pm 5.9 \\
98.8+28\end{array}$ \\
\hline
\end{tabular}

Adaptado de Kern S y colaboradores 2006 y Wagner W y colaboradores 2005.

en hueso especialmente cuando la administración de éstas células es in situ (40,41). Estudios sugieren que para garantizar la formación de hueso, es necesario el uso de materiales naturales o sintéticos (transportadores de $\mathrm{CMM}$ ), como cerámicas o titanio que son materiales inertes y osteoconductivos (3).

La diferenciación de CMM a condrocitos se ha realizado en matrices tridimensionales con colágeno (26) y con diferentes polímeros modificados como poli acrílico (PAc), poli estireno (PEs) y polietilenglicol (PEG) (27). Se ha observado que la utilización de PAc y PEs en cultivos de CMM promueven su adhesión y proliferación sin diferenciación mientras que el uso de PEG genera un efecto mayor sobre la diferenciación condrogénica.
Las CMM también juegan un papel importante en la reparación de tendón usando como transportador colágeno tipo I con distintas concentraciones autólogas de CMM (1, 4 y $8 \times 10^{6}$ células $\left./ \mathrm{ml}\right)$; obteniendo una efectividad del $30 \%$ en la reparación del tendón, lo que constituye un avance importante si se considera la complejidad del tejido recuperado (3).

En la reparación de miocardio se han postulado diferentes efectos de las CMM sobre el tejido cardiaco como la diferenciación in situ de ésta células en cardiomiocitos, liberación de factores solubles paracrinos que promuevan la proliferación de células residentes de tejido y/o la fusión de las CMM con células cardiacas. El modelo animal más usado para evaluar esta aplicación 


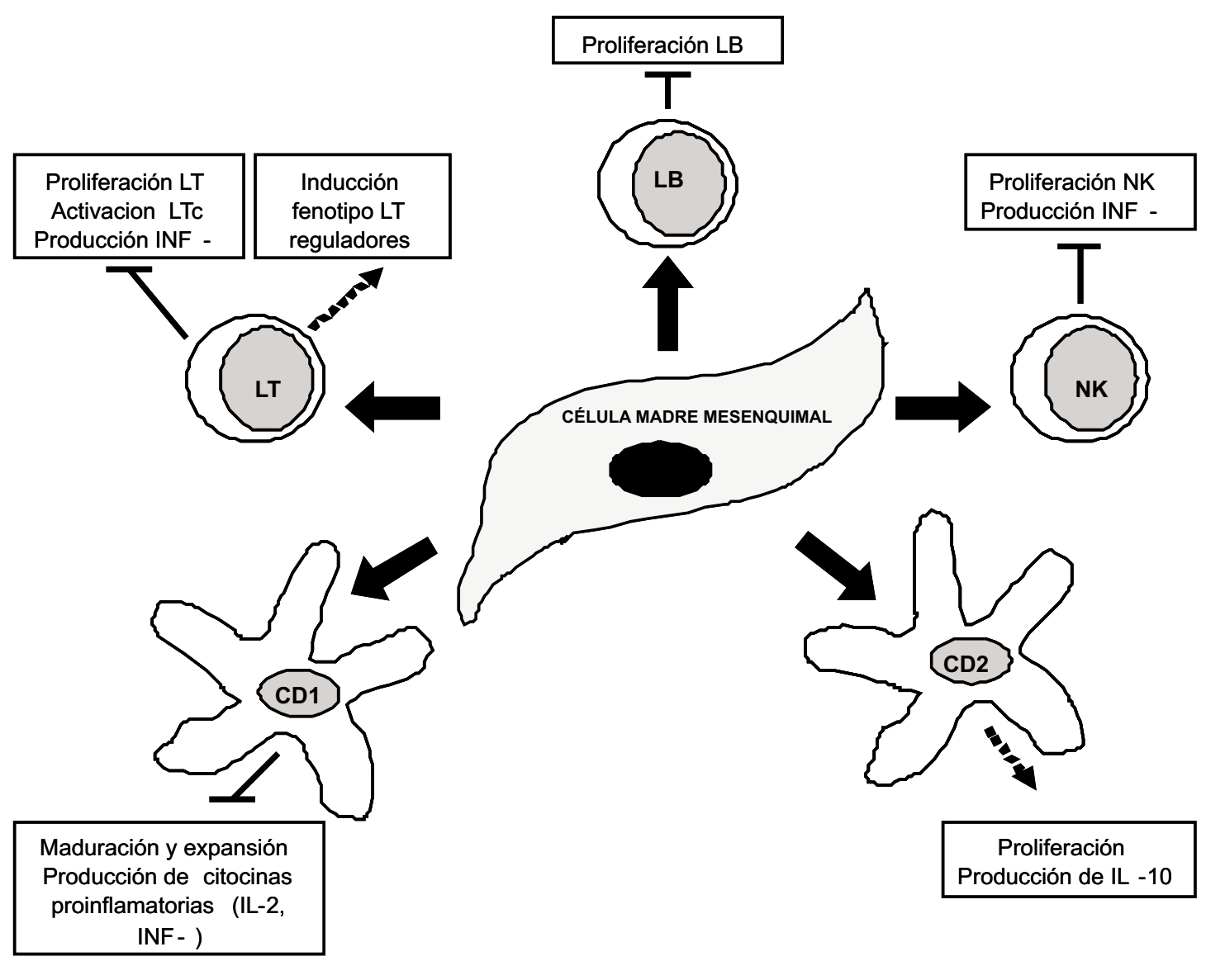

Figura 1. Efecto de las células madre mesenquimales sobre el sistema inmune. Adaptado de Rasmusson I. 2006.

es el corazón porcino donde se ha observado una reducción importante del tamaño del tejido lesionado, neovascularización y reducción de los niveles de apoptosis (42). Defectos congénitos en el músculo esquelético como distrofia muscular y otras miopatías, pueden ser teóricamente restaurados con un transplante de CMM que mejora la estructura y función del músculo. CMM obtenidas de la membrana sinovial han mostrado in vivo potencial miogénico en el modelo de ratón $m d x$ con distrofia muscular de Duchenne (43).

Además de las propiedades regenerativas de la CMM, éstas también tienen la facultad de afectar el funcionamiento del sistema inmune. Estudios muestran que las CMM pueden inhibir la proliferación de linfocitos inducida por aloantígenos (44), mitógenos como fitohemaglutinina y concavalina A (45); así como la activación a través de anticuerpos anti CD3 y CD28
(46). Durante la maduración de células dendríticas, las CMM puede inhibir la expresión de moléculas involucradas en la presentación de antigenos como CD1a, CD40, CD80, CD86 y HLA-DR (47). En co-cultivo con células mononucleares de sangre periférica, las CMM incrementan la proporción de subpoblaciones de linfocitos $\mathrm{T}$ con fenotipo de células reguladores como $\mathrm{CD} 4+/$ CD25 alto, CD4+/CTLA-4+, CD4+/CD25+/CTLA-4+ (48). En la Figura 1 se observa otros efectos de las CMM sobre células del sistema inmune (7).

Desde que las CMM fueron descritas se ha profundizado en su caracterización fenotípica, ontogénica y funcional; sin embargo, aún quedan varios aspectos por dilucidar sobre su biología especialmente en lo relacionado con su regulación a través de nichos o microambientes particulares como la médula ósea o el tejido cardiaco ya que si bien es considerada una población celular particular, 
su funcionabilidad puede variar dependiendo de la interacción directa con otras células o de la liberación de factores solubles específicos de cada microambiente; por esta razón, es importante estudiar las CMM en el contexto de nichos particulares y no generalizar su uso terapéutico sin considerar las variaciones observadas según de la fuente de obtención, número de células utilizadas y microambiente donde se desean utilizar. Una vez se avance en la identificación de los roles biológicos que llevan a cabo las CMM in vivo y los mecanismos moleculares responsables de cada una de estas funciones, la medicina regenerativa ya no considerará tratamientos temporales para enfermedades degenerativas, sino soluciones terapéuticas a largo plazo para una amplia variedad de patologías.

\section{Agradecimientos}

Al grupo de Inmunobiología y Biología Celular por el apoyo brindado a la línea de Investigación «Biología de las Células Madre» de la Facultad de Ciencias de la Pontificia Universidad Javeriana. NOV

\section{Referencias}

1. Friedenstein AJ, Deriglasova UF, Kulagina NN, Panasuk AF, Rudakowa S, Lueria E. Precursors for fibroblasts in different populations of hematopoietic cells as detected by the in vitro colony assay method. Exp Hematol. 1974;2:83 -92.

2. Beyer N, Da Silva L. Mesenchymal Stem Cells: Isolation in vitro Expansion and Characterization. Handb Exp Pharmacol. 2006; 174:249-282.

3. Krampera M, Pizzolo G, Aprili G, Franchini M. Mesenchymal Stem Cells for bone, cartilage, tendon and skeletal repair. Bone. 2006;39:678-683

4. Chang Y, Tseng C, Hsu L, Hsieh T, Hwang S. Characterization of two populations of mesenchymal progenitor cells in umbilical cord blood. Cell Biol Int. 2006;30:495-499.

5. Dominici M, Le Blanc K, Mueller I, Staper Cortenbach I, Marini F, Krause D et al. Minimal criteria for defining multipotent mesenchymal stromal cells. The International Society for Cellular Therapy position statement. Cytotherapy. 2006; 8:315-317

6. Bianco P, Riminucci M, Gronthos S, Robey P. Bone Marrow Stromal Cells: Nature, Biology, and Potencial Applications. Stem Cells. 2001;19:180-192.

7. Rasmusson I. Immune modulation by mesenchymal stem cells. Exp Cell Res. 2006;312:2169-2179.
8. Deans RJ, Moseley AB. Mesenchymal Stem Cells: Biology and potential clinical uses. Exp Hematol. 2000;28:875-884.

9. Lakshmipathy U, Verfaille C. Stem Cell Plasticity. Blood Rev. 2005; 19:29-38.

10. Rodriguez V. Células Madre: Conceptos Generales y Perspectivas de Investigación. Universitas Scientiarum. 2005;10:5-14.

11. Muraglia A, Cancedda R, Quarto R. Clonogenic mesenchymal progenitors from human bone marrow differentiate in vitro according to a hierarchical model. J Cell Sci. 2000;113:1161-1166.

12. Erices A, Conget $P$, Minguell J. Mesenchymal progenitor cells in human umbilical cord blood. Br J Haematol. 2000;109:235-242.

13. Airas L, Niemelä J, Salmi M, Puurunen T, Smith DJ, Jalkanen S. Differential Regulation and Function of CD73, a GlycosylPhosphatidylinositol-linked 70-kD Adhesion Molecule, on Lymphocytes and Endothelial Cells. J Cell Biol. 1997; 136:421-431.

14. Sträter N. Ecto- 5'-nucleotidase: Structure function relationships. Purinergic Signal. 2006;2:343-350.

15. Craig W, Kay R, Cutler RL, Landsorp PM. Expression of Thy-1 on Human Hematopoietic Progenitor Cells. J Exp Med. 1993;177:1331-1342.

16. Barboni E, Rivero BP, George AJ, Martin SR, Renoup DV, Hounsell EF et al. The glycophosphatidylinositol anchor affects the conformation of Thy-1 protein. J Cell Sci. 1995;108:487-497.

17. Wiesmann A, Buhring HJ, Mentrup C, Wiesmann HP. Decreased CD90 expression in human mesenchymal stem cells by applying mechanical stimulation. Head Face Med. 2006;31:2-8.

18. Cheifetz S, Bellon T, Cales C, Vera S, Bernabeu C, Massagué J, Letarte. Endoglin is a Component of the Transforming Growth Factor -â Receptor System in Human Endothelial Cells. J Biol Chem. 1992; 67:19027-19030.

19. Fonsatti E, Maio M. Highlights on endoglin (CD105): from basic findings towards clinical applications in human cancer. $\mathrm{J}$ Transl Med. 2004;2:18-24.

20. Roura S, Farre J, Soler-Botija C, Llach A, Hove-Madsen L, Cairo JJ et al. Effect of aging on the pluripotential capacity of human CD105 (+) mesenchymal stem cells. Eur J Heart Fail. 2006;8:555-8563.

21. Simmons PJ, Torok-Storb B. Identification of Stromal Cell Precursors in Human Bone Marrow by a Novel Monoclonal Antibody STRO-1. Blood. 1991; 8:55-62.

22. Gonçalves R, da Silva C, Cabra J, Zanjani E, Almeida-Porada G. STRO-1+ human universal stromal feeder layer to expand/maintain human bone marrow hematopoietic stem/progenitor cells in a serum-free culture system. Exp Hematol. 2006;34:1353-1359.

23. Lesley J, Hyman R, Kincade PW. Hyaluronan binding by cell surface CD44. J Biol Chem. 2000; 275:26967-26975.

24. Zhu H, Mitsuhashi N, Klein A, Barsky L, Weinberg K, Barr M et al. The Role of the Hyaluronan Receptor CD44 in Mesenchymal Stem Cell Migration in the Extracellular Matrix. Stem Cells. 2006;24:928-935.

25. Ohneda O, Ohneda K, Arai F, Lee J, Miyamoto T, Fukushima Y et al. ALCAM (CD166): Its Role in Hematopoietic and Endotelial Development. Blood. 2001; 98:2134-2142. 
26. R. Dorotkaa, U. Windbergerb, K. Macfeldab, U. Bindreitera, C. Tomaa, S. Nehrera. Repair of articular cartilage defects treated by microfracture and a three-dimensional collagen matrix. Biomaterials. 2005; 26:3617-3629.

27. Guo L, Kawazoe N, Fan Y, Ito Y, Tanaka J, Tateishi T et al. Chondrogenic differentiation of human mesenchymal stem cells on photoreactive polymer-modified surfaces. Biomaterials. 2008;29:23-32.

28. Kim J, Lee Y, Kim H, Hwang KJ, Kwon HC, Kim SK et al. Human amniotic fluid-derived stem cells have characteristics of multipotent stem cells. Cell Prolif. 2007;40:75-90.

29. Mendes S, Robin C, Dzierzak E. Mesenchymal progenitor cells localize within hematopoietic sites throughout ontogeny. Development. 2005;132:1127-1136.

30. Wexler S, Donaldson C, Denning P, Rice C, Bradley B, Hows J. Adult bone marrow is a rich source of human mesenchymal «stem» cells but umbilical cord and mobilized adult blood are not. Br J Haematol. 2003;121:368-374.

31. Baksh D, Song L, Tuan RS. Adult mesenchymal stem cells: characterization, differentiation, and application in cell and gene therapy. J Cel Mol Med. 2004; 8:301-316.

32. Wagner W, Wein F, Seckinger A, Frankhauser M, Wirkner U, Krause U et al. Comparative characteristics of mesenchymal stem cells from human bone marrow, adipose tissue, and umbilical cord blood. Exp Hematol. 2005;33:1402-1416.

33. Kern S, Eichler H, Stoeve J, Klüter H, Bieback K. Comparative Analysis of Mesenchymal Stem Cells from Bone Marrow, Umbilical Cord Blood, or Adipose Tissue. Stem Cells. 2006;24:1294-1301

34. Bieback K, Kern S, Klüter H, Eichler H. Critical parameters for the isolation of mesenchymal stem cells from umbilical cord blood. Stem Cells. 2004;22: 625-634.

35. Sabatini F, Petecchia L, Tavian M, Jodon V, Rossi GA, BroutyBoye D. Human bronchial fibroblast exhibit a mesenchymal stem cell phenotype and multilineage differentiating potentialities. Lab Invest. 2005; 85:962-971.

36. Shi S, Bartold PM, Miura M, Seo BM, Robey PG, Gronthos S. The efficacy of mesenchymal stem cells to regenerate and repair dental structures. Orthod Craniofac Res. 2005; 8:191-199.

37. Lee RH, Kim B, Choi I. Characterization and expression analysis of mesenchymal stem cells from human bone marrow and adipose tissue. Cell Physiol Biochem. 2004;14:311-324.
38. Krampera M, Pasini A, Pizzolo G, Cosmi L, Romagni S, Annunziato. Regenerative and immunomodulatory potential of mesenchymal stem cells. Curr Opin Pharmacol. 2006; 6:435-441.

39. Kassem M, Kristiansen M, Abdallah B. Mesenchymal Stem Cells: Cell Biology and Potential use in Therapy. Basic Clin Pharmacol Toxicol. 2004;95:209-214.

40. Mankani MH, Kuznetsov SA, Fowler B, Kingman A, Robey PG. In vivo bone formation by human bone marrow stromal cells: effect of carrier particle size and shape. Biotechnol Bioeng. 2001;72:96-107.

41. Tsuchida H, Hashimoto J, Crawford E, Manske P, Lou J Engineered allogeneic mesenchymal stem cells repair femoral segmental defect in rats. J Orthop Res. 2003;21:44-53.

42. Mazhari R, Hare JM. Mechanisms of action of mesenchymal stem cells in cardiac repair: potential influences on the cardiac stem cell niche. Nat Clin Pract Cardiovasc Med. 2007;4:S21-S26

43. De Bari C, Dell'Accio F, Vandenabeele F, Vermeesch JR, Raymackers JM, Luyten FP. Skeletal muscle repair by adult human mesenchymal stem cells from synovial membrane. Blood Rev. 2006; 200:161-71

44. Tse WT, Pendleton JD, Beyer WM, Egalka MC, Guinan EC. Suppresion of allogenic T-cell proliferation by human marrow stromal cells: implications in transplantation. Transplantation. 2003;75:389-397.

45. Le Blanc K, Tammik L, Sundberg B, Haynesworth SE, Ringden O. Mesenchymal stem cells inhibit and stimulate mixed lymphocyte cultures and mitogenic responses independently of the major histocompatibility complex. Scand J Immunol. 2003;57:11-20.

46. Aggarwal S, Pittenger MF. Human mesenchymal stem cells modulate allogenic immune cell responses. Blood. 2005;105:1815-1822.

47. Zhang W, Ge W, Li C, You S, Liao L, Han Q et al. Effects of mesenchymal stem cells on differentiation, maduration, and function of human monocyte-derived dendritic cells. Stem Cells Dev. 2004;13:263-3271.

48. Maccario R, Podesta M, Moretta A, Cometa A, Comoli P, Montagna $\mathrm{D}$ et al. Interaction of human mesenchymal stem cells with cells involved in alloantigen -specific immune response favors the differentiation of CD4+ T-cell subsets expressing a regulatory / suppressive phenotype. Haematologica. 2005; 90:516-525.
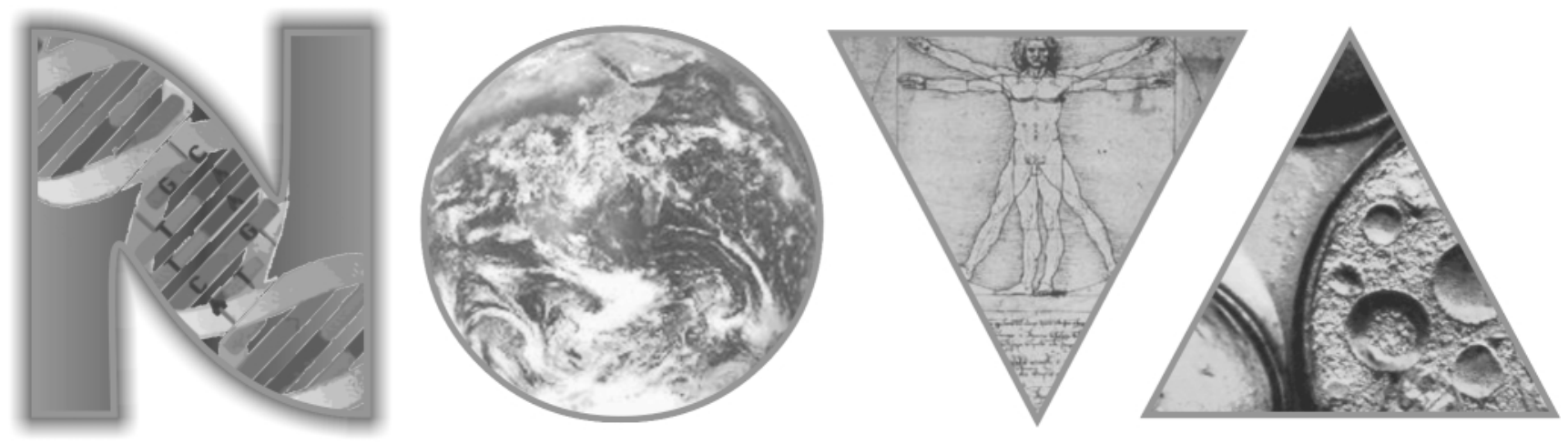